\title{
Intraoperative Echocardiographic Detection of Septal Aneurysm and Additional Ventricular Septal Defect in a Child with Tetralogy of Fallot
}

\author{
${ }^{1}$ Sambhunath Das, ${ }^{2}$ Ashok Kumar
}

\begin{abstract}
A 2-year-old child was diagnosed with subaortic ventricular septal defect (VSD) with severe infundibular and valvular pulmonary stenosis (PS) by transthoracic echocardiography. Intraoperative transesophageal echocardiography (TEE) detected aneurysmal interventricular and interatrial septum (IAS), subaortic VSD, and right ventricular outflow tract (RVOT) with an additional midmuscular VSD. The aneurysmal interventricular septum (IVS) was repaired. An accessory tricuspid papillary muscle was attached to RVOT, which was augmented with a transannular pericardial patch to have minimal postoperative gradient without sacrificing the accessory papillary muscle (APM). This rare case poses a challenge to the anesthetist and surgeon in the form of diagnostic differences, severe right ventricle (RV) dysfunction with aneurysmal IVS, and difficulty in reconstruction of RVOT. Intraoperative TEE played a greater role to diagnose the IVS aneurysm, additional VSD and guide for appropriate surgery.
\end{abstract}

Keywords: Accessory septal tricuspid papillary muscle, Interatrial septum aneurysm, Interventricular septum aneurysm, Tetralogy of fallot, Transesophageal echocardiography.

How to cite this article: Das S, Kumar A. Intraoperative Echocardiographic Detection of Septal Aneurysm and Additional Ventricular Septal Defect in a Child with Tetralogy of Fallot. J Perioper Echocardiogr 2017;5(1):21-24.

Source of support: Nil

Conflict of interest: None

\section{INTRODUCTION}

An aneurysm of IVS is a rare association with congenital cardiac defects. Most commonly, aneurysm in congenital heart diseases involves the membranous IVS. In 19\% of cases, it is associated with VSD. ${ }^{1}$ An association of IVS aneurysm in a case of Tetralogy of Fallot (TOF) was not found in the literature. Reported cases of IVS aneurysm till date were in association with acyanotic heart diseases. ${ }^{2}$ Etiology of membranous IVS aneurysm

\footnotetext{
${ }^{1}$ Professor, ${ }^{2}$ Senior Resident

1,2Department of Cardiac Anesthesia, All India Institute of Medical Sciences, New Delhi, India

Corresponding Author: Sambhunath Das, Professor Department of Cardiac Anesthesia, All India Institute of Medical Sciences, New Delhi, India, Phone: +9126593423 e-mail: dr_sambhu@yahoo.com
}

includes idiopathic, spontaneous closure of VSD, trauma, and infection. ${ }^{1}$ Muscular VSD aneurysms are common in adult patients, especially in patients with a history of myocardial infarction. Congenital muscular IVS aneurysms are reported to be associated with familial inheritance and perinatal myocardial infarction. ${ }^{1,2}$ We present our case of TOF with an incidental intraoperative detection of intact IVS aneurysm involving the muscular and membranous part and accessory tricuspid papillary muscle attached to the RVOT.

The correct diagnosis of congenital heart disease is most important to have an appropriate surgery with a better outcome. In the present era, intraoperative TEE plays a greater role to confirm the preoperative diagnosis and detect new additional findings in some cases. The present case report describes how intraoperative TEE detected the additional presence of IVS aneurysm and muscular VSD in a case of previously diagnosed case of Tetrology of Fallot.

\section{CASE REPORT}

A 2-year-old boy of $10 \mathrm{~kg}$ body weight presented with chief complaints of bluish discoloration of skin since childhood, recurrent respiratory tract infection, and history of dyspnea on exertion. The patient was diagnosed at 6 months of age with congenital heart disease and transthoracic echocardiography suggesting severe PS with confluent good sized pulmonary arteries (PAs) and subaortic VSD of $3 \mathrm{~mm}$ with the right to left shunt. Catheter angiography report 1 year earlier recorded significant collaterals from descending thoracic aorta and subaortic VSD with severe infundibular and valvular PS. The patient was planned for coiling of major aortopulmonary collateral arteries (MAPCAs) followed by total correction. Preoperative investigations showed hemoglobin of $21.5 \mathrm{~g} / \mathrm{dL}$, leukocyte count of $8,100 / \mathrm{mm}^{3}$; liver and renal function tests were within normal limits.

\section{Anesthesia Sequence}

The child was instructed for 8 hours fasting for solid food and 2 hours fasting for clear liquids prior to surgery. The patient was advised propranolol $10 \mathrm{mg}$ and promethazine 
$5 \mathrm{mg}$ orally on the morning of surgery. Operating room was prepared with anesthetic drugs, emergency drugs, and anesthesia machine checked. Fentanyl $20 \mu \mathrm{g}$, ketamine $20 \mathrm{mg}$, and rocuronium $10 \mathrm{mg}$ were given for induction. The patient was intubated with $5.0 \mathrm{~mm}$ uncuffed endotracheal tube fixed at $12 \mathrm{~cm}$. Anesthesia was maintained with sevoflurane and an intermittent bolus dose of rocuronium. Central venous catheter was placed in a right femoral vein and arterial line placed in the left femoral artery. Baseline arterial blood gases showed metabolic acidosis with $\mathrm{pH}$ of 7.27 and -10 base excess that was corrected with $\mathrm{NAHCO}_{3}$.

\section{Transesophageal Echocardiography Findings}

Intraoperative TEE (Philips iE33 ultrasound machine, pediatric 57 esophageal transducer) and midesophageal (ME) four-chamber view revealed very thin aneurysmal IVS with paradoxical movement toward the left ventricle (LV) (Fig. 1). Both muscular and membranous IVS

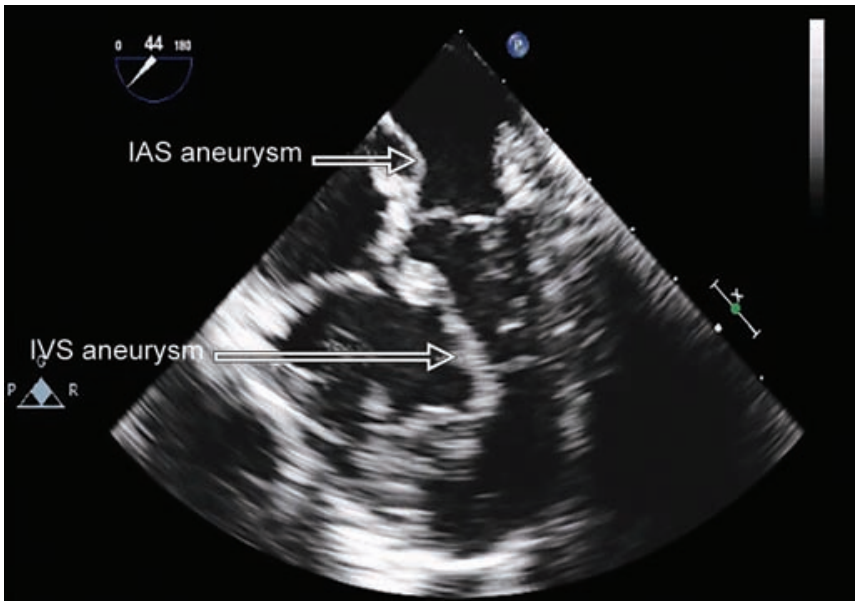

Fig. 1: Transesophageal echocardiography midesophageal fourchamber view with aneurysmal IVS and IAS (marked by arrow)

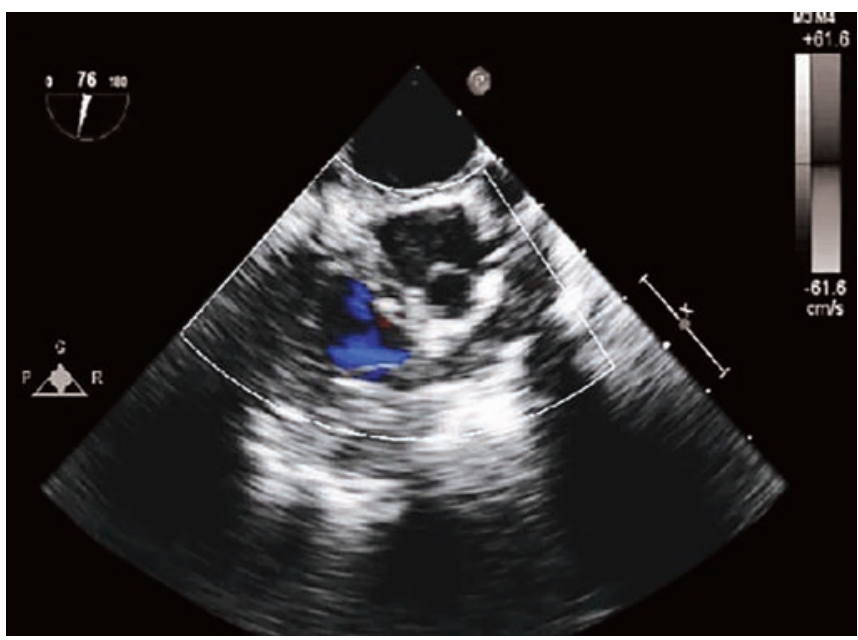

Fig. 3: Transesophageal echocardiography midesophageal RV inflow-outflow view, severe PS was noted at valvular and RVOT appeared aneurysmal. A 3-mm size midmuscular VSD and a subaortic 3-mm size VSD were detected (Fig. 2). Tricuspid valve (TV) appeared to be normal, RV dilated and hypertrophied, and LV appeared small in size. In ME RV inflow outflow view, severe PS was noted with valvular and infundibular type (Fig. 3). Patent foramen ovale was found and IAS appeared aneurysmal (Fig. 1). An ME aortic long axis (LAX) view showed small LV with dilated RV and aneurysmal IVS (Fig. 4). In transgastric RV outflow view, PS gradient was measured as $51 \mathrm{~mm} \mathrm{Hg}$.

\section{Surgical Sequence and Operative Findings}

After midline sternotomy and systemic heparinization with $400 \mathrm{IU} / \mathrm{kg}$, aortic and bicaval venous cannulation was performed to initiate cardiopulmonary bypass (CPB). The right atrium (RA) was opened, IAS was aneurysmal, but no intervention was required. Subaortic and midmuscular VSD (Fig. 5) were closed by direct sutures from the trans-RA approach. The IVS was

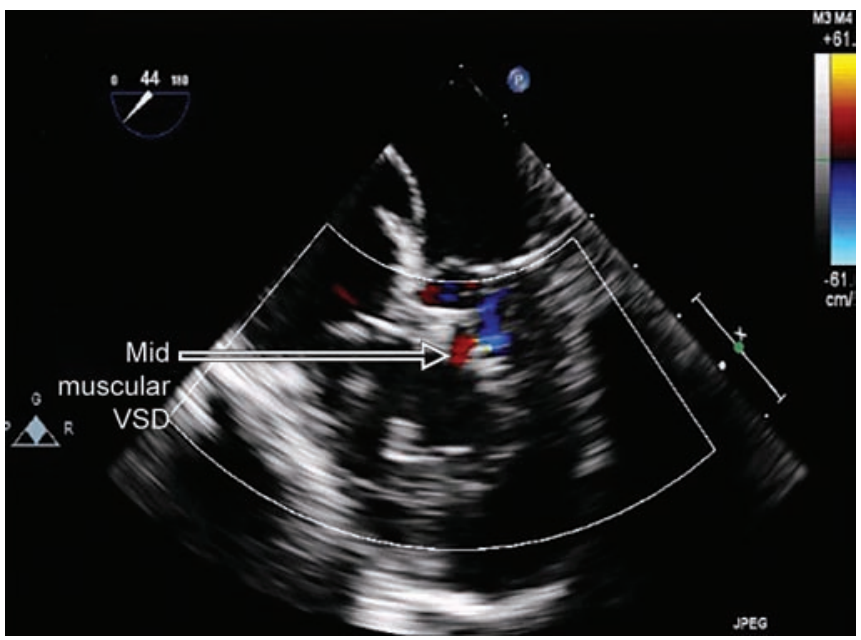

Fig. 2: Transesophageal echocardiography midesophageal fourchamber view with midmuscular VSD with right to left shunt

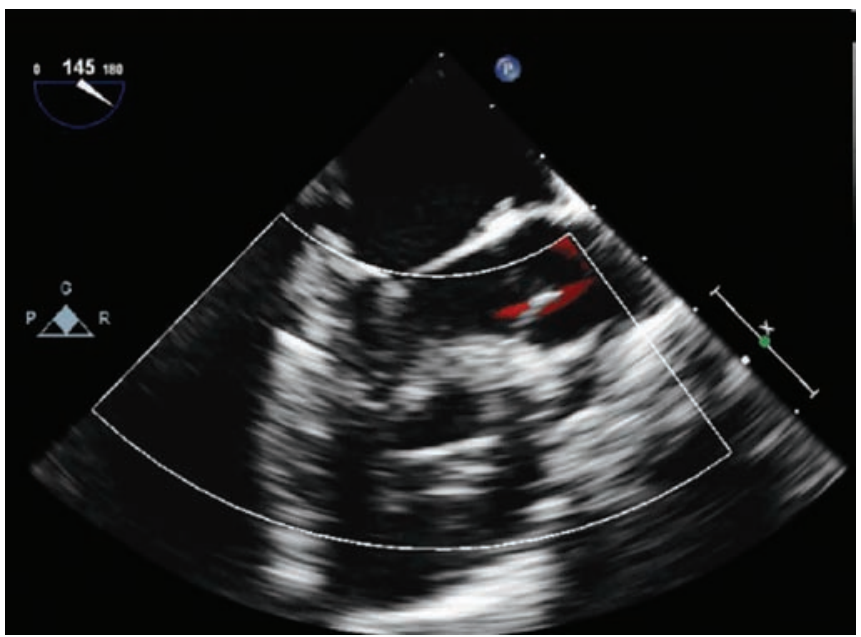

Fig. 4: Transesophageal echocardiography midesophageal LAX view with small LV, IVS aneurysm, and dilated RV 
aneurysmal and plicated with Teflon felt-supported sutures. A transannular incision was given at RV outflow to proximal part of the main PA. Infundibular resection was attempted. An APM was attached from RVOT to septal tricuspid leaflet of TV. The APM was not resected; because unbalanced support to TV leaflets would have caused tricuspid regurgitation. The pericardial patch was placed at the transannular region to dilate RVOT (Fig. 6). During rewarming phase of $\mathrm{CPB}$, sodium nitroprusside $0.5 \mu \mathrm{g} / \mathrm{kg} / \mathrm{min}$ and dobutamine $5 \mu \mathrm{g} / \mathrm{kg} / \mathrm{min}$ were started. After TEE-guided deairing of cardiac chambers, correcting electrolyte imbalance, resuming electrical activity of heart, and adequate ejection of RV and LV, the patient was weaned from CPB. Total CPB time was 163 minutes. Heparin was reversed with protamine.

\section{Postoperative Transesophageal Echocardiography}

Postrepair ME four-chamber view showed decrease in RA and RV size from baseline. No residual VSD was seen
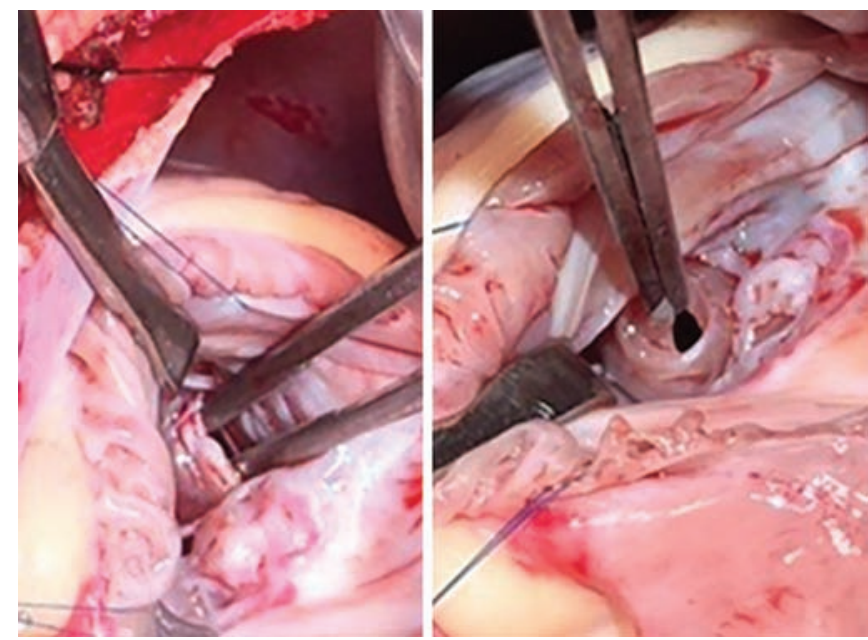

Fig. 5: Intraoperative view on left half showing subaortic VSD with forceps inserted in it. Image on right half shows midmuscular VSD held by forceps

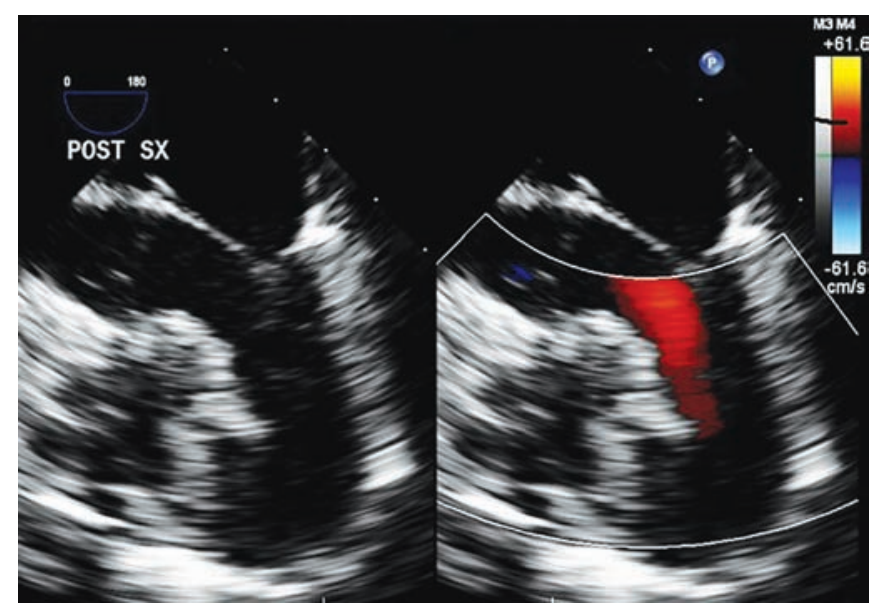

Fig. 7: Postoperative TEE midesophageal five-chamber view showing no residual VSD in subaortic and midmuscular region
(Fig. 7). An IVS was aneurysmal with paradoxical movement toward LV during systole. Patent foramen ovale was left open. Flow across pulmonary valve was adequate. The ME/RV inflow-outflow view showed adequate patency at infundibular region (Fig. 8). Transgastric RV outflow view measured RVOT peak gradient of $10 \mathrm{~mm} \mathrm{Hg}$.

The patient was shifted to intensive care unit for elective mechanical ventilation. Inotropes were tapered gradually as per stability of the vital parameters. The patient was gradually weaned from ventilation and extubated on postoperative day 2. No postoperative complications were observed.

\section{DISCUSSION}

Aneurysm of IVS in congenital heart disease is a rare complication. Studies suggest most of these aneurysms originated in membranous IVS, as it is the weakest portion of the septum. ${ }^{1}$ Data on muscular IVS aneurysms are very

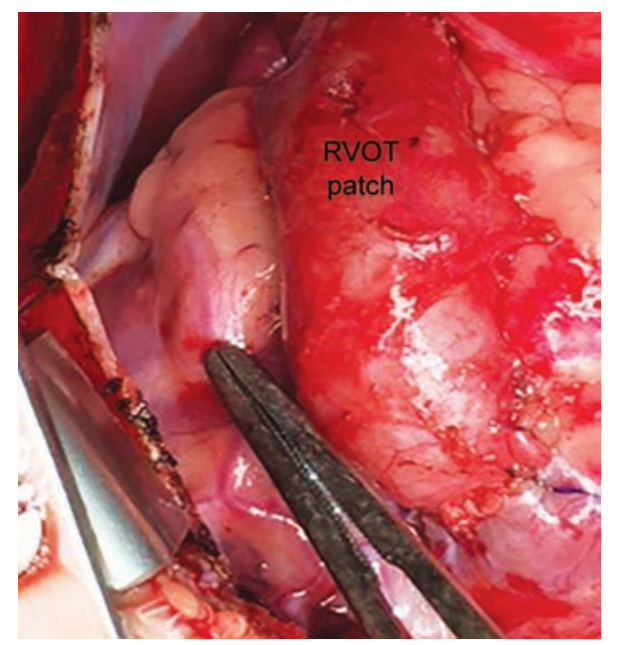

Fig. 6: Intraoperative image with pericardial patch applied on RVOT

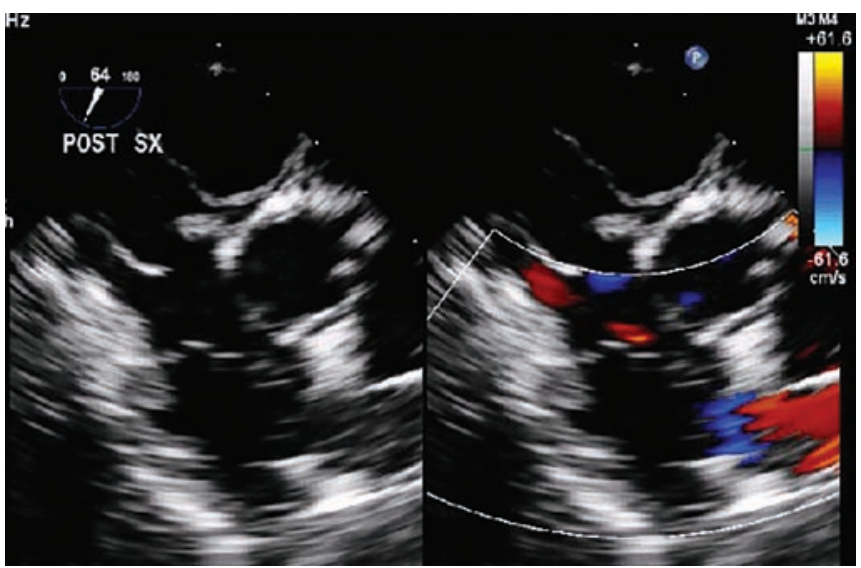

Fig. 8: Postoperative TEE RV inflow-outflow showing adequate unobstructive flow in infundibular region 
limited. Our case had both membranous and muscular IVS aneurysm, there was severe infundibular and valvular PS, with subaortic and midmuscular VSD which were causing right to left shunt. The size of VSD was so small that it had pressure effects on right side chambers, resulting in dilatation of RV and aneurysm of IVS. Left side chambers were reduced in size by a septal aneurysm and IVS was thinned out. Free wall of RV was hypertrophied and IVS was moving paradoxically toward LV during systole.

Patients with IVS aneurysm are mostly asymptomatic or may produce serious hemodynamic disturbances due to infection, rupture, and obstruction. ${ }^{3}$ The most common lesions associated are aortic insufficiency, subaortic stenosis, and conduction abnormalities. ${ }^{4}$ No aortic insufficiency or stenosis was present in our case. Diagnosis of IVS aneurysm has been reported to be possible in intrauterine echocardiography. ${ }^{5}$ The aneurysm is mostly involved in the membranous part of IVS and may create defect in atrioventricular valves. ${ }^{5}$ Frequent complications are rupture of aneurysm, subacute bacterial endocarditis, thromboembolism, and obstruction of the RVOT. ${ }^{3}$ Patient presentation depends on many factors like size of VSD, severity of PS, direction of shunt, amount of hypertrophic RV, and MAPCA. Common complaints of TOF with IVS aneurysm are central cyanosis, failure to thrive, and dyspnea on exertion due to RV failure. Oxygenation depends on size and number of collaterals and severity of RVOT obstruction. Isolated IVS aneurysm with hypertrophied RV free wall is a rare case and reason for this is unknown. In our case patient also had IAS aneurysm with patent foramen ovale.

The decision of surgical repair of congenital IVS aneurysm is a matter of discrepancy for surgeons. An IVS was repaired by plication and shortening of septum in the present case. We were thoughtful that after resection of RVOT obstruction the septum will regain and remodel to normal size and shape. After IVS placation, possibility of conduction tissue damage leading to conduction block or arrhythmias can occur ${ }^{6}$ but was not observed in our case. We had unanticipated difficulty in the form of accessory tricuspid papillary muscle arising from RVOT. There were two choices for relieving RVOT obstruction, resection over no-resection of the APM of TV. Resection of APM might have caused tricuspid insufficiency. As transannular patch relieved the RVOT obstruction after surgery, we did not want to resect accessory tricuspid papillary muscle.
There were no case reports with similar cardiac morphology; this case gives an example of how important is preoperative diagnosis. Intraoperative TEE is useful in the present case for detecting aneurysmal IVS and IAS with additional muscular VSD. In our case, midmuscular VSD was not detected in transthoracic echocardiography and catheter angiography. The TEE helped to assess the adequate repair of RVOT stenosis by measuring gradient across the PV. Intraoperative TEE had an important role in our case for complete diagnosis and subsequent management with successful outcome.

\section{CONCLUSION}

The present case gives a new challenge to echocardiographers, anesthetists, and surgeons to have an exact diagnosis in complex congenital heart disease. Intraoperative echocardiography detected aneurysm of IVS and IAS along with additional muscular VSD in this unusual rare case of TOF. Transesophageal echocardiography was useful to guide the surgical repair, resulting in a successful postoperative outcome.

\section{REFERENCES}

1. Nguyen TP, Srivastava S, Ko HH, Lai WW. Congenital muscular ventricular septal aneurysm: report of four cases and review of the literature. Pediatr Cardiol 2008 Jan;29(1): 40-44.

2. Eriksson H, Cooper SM, Rosenbaum KN, Ruckman RN. Familial occurrence of congenital aneurysm of the muscular interventricular septum. Pediatr Cardiol 1998 May-Jun; 19(3):249-252.

3. Jian-Jun G, Xue-Gong S, Ru-Yuan Z, Min L, Sheng-Lin G, Shi-Bing Z, Qing-Yun G. Ventricular septal defect closure in right coronary cusp prolapse and aortic regurgitation complicating VSD in the outlet septum: which treatment is most appropriate? Heart Lung Circ 2006 Jun;15(3):168-171.

4. Ghosh S, Ghosh AK, Ghosh SK. Patent foramen ovale and atrial septal aneurysm in cryptogenic stroke. Postgrad Med J 2007 Mar;83(977):173-177.

5. Espinoza J,KalacheK, Gonçalves LF, LeeW,Chaiworapongsa T, Schoen ML, Devers P, Treadwell M, Mazor M, Romero R. Prenatal diagnosis of membranous ventricular septal aneurysms and their association with absence of atrioventricular valve 'offsetting'. Ultrasound Obstet Gynecol 2004 Dec;24(7):787-792.

6. Perrotta S, Guarini P, Accadia M, Canciello M, Spadaro P, Ruta A, Gallotto V, Granato Corigliano G. Atrial septal aneurysm. Report of two clinical cases and review of the literature. Minerva Cardioangiol 1995 Jul-Aug;43(7-8):339-343. 